\title{
THE ALMOST ISOMORPHISM RELATION FOR SIMPLE REGULAR RINGS
}

\author{
PERE ARA AND K. R. GoodearL
}

Dedicat a la memòria del nostre amic, Pere Menal i Brufal

\begin{abstract}
$\Lambda$ longstanding open problem in the theory of von Neumann regular rings is the question of whether every directly finite simple regular ring must be unit-regular. Recent work on this problem has been done by P. Menal, K.C. O'Meara, and the authors. To clarify some aspects of these new developments, we introduce and study the notion of almost isomorphism between finitely generated projective modules over a simple regular ring.
\end{abstract}

\section{0 . Introduction.}

In the few past years, there have becn some advances in the understanding of directly finite simple regular rings. In 1988, Menal and the second author [GM, Theorem 5.2] showed that if $R$ is a directly finite regular algebra over an uncountable field, and if $R$ contains no uncountable direct sums of nonzero right ideals, then it is unit-regular. As a consequence of this, any stably finite simple regular algebra $R$ over an uncountable field is unit-regular [GM, Corollary 5.4]. More recently, O'Meara proved that a directly finite simple regular ring satisfying weak comparability is unit-regular [O, Theorem 1]. An alternative proof of O'Meara's 'Theorem was developed by the second author in privately circulated notes [G5]. We take the opportunity to present this proof here.

Our standard reference for the theory of regular rings is [G1], and for the theory of partially ordered abelian groups is [G4]. The reader can refer to these books for any undefined terms.

The research of the first author was partially supported by DGICY'T grant P89-0296, and that of the second author by an NSF grant 
Let $R$ be an associative ring with 1 . Denote by $\mathcal{P}$ (resp. $\mathcal{P}_{0}$ ) the class of finitely generated projective right $R$-modules (resp. the class of nonzero finitely generated projective right $R$-modules). If $R$ is a regular ring then $L\left(R_{R}\right)$ will denote the lattice of principal right ideals of $R$. For $A, B \in \mathcal{P}$, we will write $A \leq B$ (resp. $A \prec B$ ) if $A$ is isomorphic to a submodule (resp. proper submodule) of $B$. For a positive integer $k$ and module $A$, we let $k A$ denote the direct sum of $k$ copics of $A$.

A ring $R$ is said to be directly finite if $x y=1$ implies $y x=1$, for $x, y \in R$. We say that $R$ is stably finite if $M_{n}(R)$ is directly finite for all $n \geq 1$. It is not known whether directly finite regular rings are stably finite [G1, Open Problem 1]. The question is open even in the case of simple regular rings.

A ring $R$ is said to be unit-regular if for any $x \in R$ there exists a unit $u \in R$ such that $x=x u x$. Every unit-regular ring is stably finite, but there exist stably finite regular rings which are not unit-regular [G1, Proposition 5.2 and Example 5.10]. However, there are some interesting classes of regular rings for which it is known that direct finiteness implies unit-rcgularity. For example, this holds for regular rings satisfying general comparability [G1, Theorem 8.12], for right $\aleph_{0}$-continuous regular rings [G2, Theorem 1.4], and for $\aleph_{0}$-complete regular rings [Bu, Corollary 1.6]. An outstanding question in the theory is whether a dircctly finite simple regular ring is unit-regular [G1, Open Problem 3].

We say that a class of modules $\mathcal{C}$ satisfies the concellation property (with respect to the isomorphism relation) if $A \oplus C \cong B \oplus C$ implies $A \cong B$ for $A, B, C \in \mathcal{C}$. A regular ring $R$ is unit-regular if and only if $\mathcal{P}$ satisfies the cancellation property, see [G1, Theorem 4.5]. The main result of Section 1 states that a directly finite simple regular ring is unitregular if and only if $\mathcal{P}$ satisfies the cancellation property with respect to the almost isomorphism relation (defined in Section 1 ).

We say that $R$ is strictly unperforated whonever $n A \prec n B$ implies $A \prec B$ for $A, B \in \mathcal{P}$ and $n \geq 1 . R$ is unperforoted if $n A \lesssim n B$ implies $A \lesssim B$ for $A, B \in \mathcal{P}$ and $n \geq 1$.

Assume that $R$ is a directly finite simple regular ring. It is an open question whether $R$ is (strictly) unperforated. Strictly unperforated directly finite simple regular rings have a number of interesting properties. In particular they are unit-regular and, in the non-artinian case, they are close to being rings of matrices of any size (see Section 2). Some technical results needed to obtain the latter statement are included in an Appendix.

Let $R$ be a stably finite simple regular ring. Then $\left(K_{0}(R),[R]\right)$ is a partially ordered abclian group with order-unit, sec [G1, Proposition 
15.3]. Let $\Phi: K_{0}(R) \longrightarrow \operatorname{Aff}\left(S\left(K_{0}(R),[R]\right)\right)$ be the natural map, see [G4, Chapter 7]. For any compact convex set $S$, denote the strict ordering on $\mathrm{Aff}(S)$ by $\ll$, that is, $f \ll g$ if and only if $f(x)<g(x)$ for all $x \in S$. By [B3, Theorem 3.1.4] and [G4, Theorem 4.12], $R$ is strictly unperforated if and only if $\Phi([A]) \ll \Phi([D])$ implies $A \prec D$ for $A, D \in \mathcal{P}$. We can consider the following weaker condition:

For any $D \in L\left(R_{R}\right)$, there cxists $K \geq 1$ such that, for $A \in L\left(R_{R}\right)$, if $K \Phi(|A|) \ll \Phi([D])$ then $A \prec D$.

We will see in Section 3 that $R$ satisfies this condition if and only if $R$ satisfies the following property:

The doubling condition $(D D)$ : For any $D \in L\left(R_{R}\right)$, there exists $K \geq 1$ such that, for $A \in L\left(R_{R}\right)$, if $A \leq D$ and $K \Phi([A]) \ll \Phi([D])$ then $2 A \prec D$.

Similarly, the following two conditions are equivalent for a directly finite simple regular ring $R$ :

Weak comparability (O'Meara): For any $D \in L\left(R_{R}\right)$ there exists $n \geq 1$ such that, for $A \in L\left(R_{R}\right)$, if $n A \lesssim R$ then $A \lesssim D$.

(dd): For any $D \in L\left(R_{R}\right)$ there exists $n \geq 1$ such that, for $A \in L\left(R_{R}\right)$, if $A \leq D$ and $n A \leqq R$ then $2 A \leqq D$.

Observe, in particular, that the doubling condition implies weak comparability in any stably finite simple regular ring.

We close the paper by studying the effect of imposing comparability with respect to the (approximately) almost isomorphism rclation on a stably finite simple regular ring.

\section{Stable range of simple regular rings.}

In this Section we study the stable range of simple regular rings, obtaining a restriction on the behaviour of the stable range on the family of finitely generated projective modules. It is easy to show by using our results that if there exists a simple regular ring of stable range 2 , then there are corner rings of $R$ with arbitrary finite stable range $n>1$. So, the situation for simple regular rings differs very much from the situation for arbitrary regular rings, see [MM; GMM].

We will apply the results on stable range to give the new proof of O'Meara's Thcorem [O, Theorem 1].

Recall that a ring $R$ satisfies the n-stable range condition (for a given positive integer $n$ ) if whenever $a_{1}, \ldots, a_{n+1} \in R$ with $a_{1} R+\cdots+a_{n+1} R=$ $R$, there exist elements $b_{1}, \ldots, b_{n} \in R$ such that

$$
\left(a_{1}+a_{n+1} b_{1}\right) R+\cdots+\left(a_{n}+a_{n+1} b_{n}\right) R=R .
$$


If $n$ is the least positive integer such that $R$ satisfies the $n$-stable range condition, then $R$ is said to have stable range $n$, and we write $\operatorname{sr}(R)=n$. It is well-known that a regular ring has stable range one if and only if it is unit-regular [G1, Proposition 4.12]. The reader is referred to [V] for the basic properties of the stable range and to $[\mathbf{W} ; \mathbf{M M} \mathbf{M}]$ for the connections between cancellation properties of modules and the stable range of their endomorphism rings.

Lemma 1.1. Let $R$ be a non-artinian simple regular ring. Then for each $P \in \mathcal{P}_{0}$ and for all $k \geq 1$, there exists $Q \in \mathcal{P}_{0}$ such that $k Q \lesssim P$.

Proof: Clearly we can assume that $P=e R$ for some nonzero idempotent $e \in R$. Since $R$ is not artinian, $e R=e_{1} R \oplus e_{2} R$ for some nonzero idempotents $e_{1}, e_{2}$. Since $R$ is simple, $e_{1} R \leqslant n\left(e_{2} R\right)$ for some $n$ and so $e_{1} R=A_{1} \oplus \cdots \oplus A_{n}$ with $A_{i} \leqq e_{2} R$ by [G1, Corollary 2.9]. Then $e R=A_{1} \oplus\left(A_{2} \oplus \cdots \oplus A_{n} \oplus e_{2} R\right)$ and clearly $2 A_{1} \lesssim e R$. Now, the result follows by induction.

The following result is patterned after an argument of Rieffel [R].

Theorem 1.2. Let $R$ be a simple regular ring such that $\operatorname{sr}(e R e) \leq k$ for some $k \geq 1$ and all idempotents $e \in R$. Then $R$ is unit-regulor.

Proof: If $R$ is artinian, the result is well-known. So we can restrict ourselves to the non-artinian case. Assume that $P_{1} \oplus n R \cong P_{2} \oplus n R$ for some finitely generated projective modules $P_{1}$ and $P_{2}$. If $P_{1}=P_{2}=0$, we are done, so we can assume that $P_{1} \neq 0$. By Lemma 1.1, $P_{1} \cong k Q \oplus U$ for some $Q \in \mathcal{P}_{0}$, and clearly we can assume that $Q \cong e R$ for some idempotent $e \in R$. Now, we have

$$
k Q \oplus U \oplus n R \cong P_{2} \oplus n R .
$$

Since $R$ is simple we have $R \oplus V \cong s Q$ for some $s \geq 1$ and since $k Q \oplus$ $U \oplus n R \oplus n V \cong P_{2} \oplus n R \oplus n V$ we have $k Q \oplus U \oplus n s Q \cong P_{2} \oplus n s Q$. By [W, Theorem 1.2], $k Q \oplus U \cong P_{2}$ and so $P_{1} \cong P_{2}$. By [G1, Theorem 4.5], it follows that $R$ is unit-regular.

We need the essentially-known fact that the finiteness of the stable range is Morita-invariant. We include a proof of this result, which is a straightforward application of the techniques in $[\mathbf{W}]$.

Lemma 1.3. Let $P$ and $Q$ be finitely generated projective modules over a ring $R$. Assume that there exists $k \geq 1$ such that $Q \oplus U \cong k P$ and there exists $i \geq 1$ such that $P \oplus T \cong i Q$, that is, $P$ and $Q$ generate 
the same categories of modules. Then $\operatorname{sr}\left(\operatorname{End}_{R}(P)\right)<\infty$ if and only if $\operatorname{sr}\left(\operatorname{End}_{R}(Q)\right)<\infty$.

Proof: We will follow the proof in [W, Theorem 1.11]. Assume that $\operatorname{sr}\left(\operatorname{End}_{R}(Q)\right)=r<\infty$. Set $m=(r+i-1) k$. Let

$$
N=P_{\mathfrak{l}} \oplus K=P_{1}^{\prime} \oplus \cdots \oplus P_{m}^{\prime} \oplus L .
$$

where $P_{1} \cong P_{j}^{\prime} \cong P$ for all $j$. Adding $T$ to this relation we obtain

$$
M:=N \oplus T=Q_{1} \oplus \cdots \oplus Q_{i} \oplus K=Q_{1}^{\prime} \oplus \cdots \ominus Q_{z+r-1}^{\prime} \oplus V \oplus L
$$

where $Q_{p} \cong Q_{q}^{\prime} \cong Q$ for all $p, q$. Applying [W, Theorem 1.11], we get a submodule $B$ of $M$ such that

$$
M=B \oplus K=B \oplus C \oplus L
$$

where $C \subseteq Q_{1}^{\prime} \oplus \cdots \oplus Q_{i+r-1}^{\prime} \oplus V$. Now we have

$$
N=(B \cap N) \oplus K=[(B \oplus C) \cap N] \oplus L
$$

and also $(B \oplus C) \cap N=(B \cap N) \ominus[(B \oplus C) \cap K]$. Let $\pi$ be the projection of $N$ onto $P_{1}^{\prime} \oplus \cdots \oplus P_{m}^{\prime}$ along $L$. Then

$$
[(B \oplus C) \cap K] \oplus L=\pi((B \oplus C) \cap K) \oplus L .
$$

It follows that $N=(B \cap N) \oplus \pi((B \oplus C) \cap K) \oplus L$. By [W, Theorem 1.6], $\operatorname{sr}\left(\operatorname{End}_{R}(P)\right) \leq m<\infty$.

Remark 1.4. If $\operatorname{sr}\left(\operatorname{End}_{R}(Q)\right)=r$ then by the above proof we obtain the following bound: $\operatorname{sr}\left(\operatorname{End}_{R}(P)\right) \leq(r+i-1) k$. In particular, if $e$ is an idempotent of $R$ and $R \leq n(e R)$ then $\operatorname{sr}(R) \leq s r(e R e)+n-1$. This is exactly the same bound obtained by Blackadar for $C^{*}$-algcbras, see [B1, Lemma A6], [B2, p.33].

Our following result is an immediate consequence of Theorem 1.2 and Lemma 1.3.

Theorem 1.5. Let $R$ be a simple regular ring. Then one of the following possibilities occurs:

(1) $R$ is unit-regular.

(2) $\operatorname{sr}\left(\operatorname{End}_{R}(P)\right)=\infty$ for every $P \in \mathcal{P}_{0}$.

(3) $\operatorname{sr}\left(\operatorname{End}_{R}(P)\right)$ is finite for every $P \in \mathcal{P}$ and the set $\left\{\operatorname{sr}\left(\operatorname{End}_{R}(P)\right) \mid\right.$ $P \in \mathcal{P}\}$ is not bounded. 
Remark 1.6. Let $R$ be any simple ring which is not stably finite. By simplicity, we then have $2 n R \leqq n R$ for some $n \geq 1$. By [ $\mathbf{W}$, Theorem 1.2], $\operatorname{sr}\left(\right.$ End $\left._{R}(n R)\right)=\infty$. By Lemma 1.3, this implies that $s r\left(\operatorname{End}_{R}(P)\right)=\infty$ for every nonzero finitely generated projective $\mathrm{R}$ module.

Theorem 1.7. Let $R$ be a simple regular ring. Assume that whenever $B, C_{1}, C_{2} \in L\left(R_{R}\right)$ with $R \oplus B \lesssim R \oplus C_{i}$ for $i=1,2$, then $B \lesssim C_{1} \oplus C_{2}$. Then $R$ is unit-regular.

Proof: Since it is easily seen that the hypothesis is inherited by the corner rings of $R$, it suffices by Theorem 1.2 to show that $\operatorname{sr}(R) \leq 2$. Let $a, b, c \in R$ such that $a R+b R+c R=R$. There is an idempotent $e \in R$ such that $c R=e R \oplus[c R \cap(a R+b R)]$. Note that $e=c t$ for some $t \in R$, and $R=(a R+b R) \oplus e R$.

Now $R_{R}=C_{1} \oplus D_{1}=C_{2} \oplus D_{2}$ where $C_{1}=\operatorname{rann}_{R}(a)$ and $C_{2}=$ r.ann $R_{R}(b)$. We observe that left multiplication by $a$ induces an isomorphism of $D_{1}$ onto $a R$, and similarly $D_{2} \cong b R$. By using this we see that $R \oplus e R \lesssim R \oplus C_{i}$ for $i=1,2$. By our hypothesis, we deduce that $e R \leqq C_{1} \oplus C_{2}$, so $e R=E_{1} \oplus E_{2}$ with each $E_{i} \lesssim C_{i}$.

Define $x \in R$ such that $x D_{1}=0$ and $x R=x C_{1}=E_{1}$; note that $x=e x$. Since $a C_{1}=0$ and $a D_{1}=a R$, we get $(a+x) R=a R+E_{1}$. Likewise, there exists $y \in e R$ such that $(b+y) R=b R+E_{2}$. Then we have $(a+c t x) R+(b+c t y) R=(a+x) R+(b+y) R=a R+b R+E_{1}+E_{2}=$ $a R+b R+e R=R$. This shows that $\operatorname{sr}(R) \leq 2$.

We now introduce a key concept for this paper, namely the almost isomorphism relation.

Definitions. Let $R$ be a regular ring and let $A, B \in \mathcal{P}$.

We say that $A$ is almost subisomorphic to $B$, written $A \leqslant_{a} B$, if for all nonzero $C \in L\left(R_{R}\right)$ we have $A \lesssim B \oplus C$. We say that $A$ is almost isomorphic to $B$, written $A \cong_{a} B$, if $A \coprod_{a} B$ and $B \coprod_{a} A$.

We say that $A$ is approximately almost subisomorphic to $B$, written $A \lesssim_{a a} B$, if for all nonzero $C \in L\left(R_{R}\right)$ there exists $n \geq 1$ such that $n A \lesssim n(B \oplus C)$. We say that $A$ is approximately almost isomorphic to $B$, written $A \cong_{a a} B$, if $A \coprod_{a a} B$ and $B \lesssim_{a a} A$.

The above notions are specially useful when $R$ is a simple regular ring which is not artinian. Since artinian simple regular rings are trivial for our theory, we will frequently assume that our simple regular rings are not artinian.

Lemma 1.8. Let $R$ be a non-artinian simple regular ring and let 
$A, B \in P$. Then:

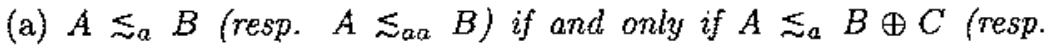
$\left.A \leqslant_{a a} B \oplus C\right)$ for all nonzero $C \in L\left(R_{R}\right)$.

(b) The relation $\Sigma_{a}$ (resp. $\leqslant_{a a}$ ) is transitive.

Proof: (a) Assume that $A \leqslant_{a} B \oplus C$ for all nonzero $C \in L\left(R_{R}\right)$. Fix a nonzero $D \in L\left(R_{R}\right)$. Then since $R$ is not artinian $D=D_{1} \oplus D_{2}$ for nonzero $D_{1}, D_{2} \in L\left(R_{R}\right)$. So $A \lesssim\left(B \oplus D_{1}\right) \oplus D_{2}=B \oplus D$. Thus $A \lesssim_{a} B$. The other implication is trivial. The proof for the relation $\lesssim_{a a}$ is analogous. (b) is proved in a similar way.

Theorem 1.9. Let $R$ be a directly finite simple regular ring. Then the following conditions are equivalent:

(a) For all $A, B, C \in \mathcal{P}, A \oplus B \S_{a} A \oplus C$ implies $B \lesssim_{a} C$.

(b) For all $B, C \in L\left(R_{R}\right), R \oplus B \leq R \oplus C$ implies $B \leqslant_{a} C$.

(c) $R$ is unit-regular.

Proof: (a) $\Rightarrow$ (b): This is clear.

(b) $\Rightarrow$ (c): Apply Theorem 1.7 .

(c) $\Rightarrow$ (a): This is immediate from the cancellation property of unitregular rings.

Lemma 1.10. Let $A, B, C$ be finitely generated projective right modules over a regular ring, such that $A \oplus C \cong B \oplus C$. Let $n \in \mathrm{N}$. Then there exist decompositions $A=A^{\prime} \oplus A^{\prime \prime}$ and $B=B^{\prime} \oplus B^{\prime \prime}$ and $C=C^{\prime} \oplus C^{\prime \prime}$ such that $A^{\prime} \cong B^{\prime}$ and $A^{\prime \prime} \oplus C^{\prime \prime} \cong B^{\prime \prime} \oplus C^{\prime \prime}$, and also $n\left(A^{\prime \prime} \oplus B^{\prime \prime}\right) \leqq C^{\prime}$.

Proof: By [G3, Lemma 2.2], there are decompositions $A=A_{11} \oplus A_{12}$ and $B=B_{11} \oplus B_{12}$ and $C=C_{11} \oplus C_{12}$ such that $A_{11} \cong B_{11}$ and $A_{12} \oplus C_{12} \cong B_{12} \oplus C_{12}$, while also $A_{12} \cong C_{11}$. Applying this lemma repeatedly, we obtain decompositions $A_{i-1,2}=A_{i 1} \oplus A_{i 2}$ and $B_{i-1,2}=$ $B_{i 1} \oplus B_{i 2}$ and $C_{i-1,2}=C_{i 1} \oplus C_{i 2}$, for $i=2,3, \ldots$, such that $A_{i 1} \cong B_{i 1}$ and $A_{i 2} \oplus C_{i 2} \cong B_{i 2} \oplus C_{i 2}$, while also $A_{i 2} \cong C_{i 1}$.

Now set $A_{1}=A_{11} \oplus A_{21} \oplus \cdots \oplus A_{n 1}$ and $A_{2}=A_{n 2}$, and define $B_{1}, B_{2}$, $C_{1}, C_{2}$ similarly. Then $A=A_{1} \oplus A_{2}$ and $B=B_{1} \oplus B_{2}$ and $C=C_{1} \oplus C_{2}$, with $A_{1} \cong B_{1}$ and $A_{2} \oplus C_{2} \cong B_{2} \oplus C_{2}$. Since $A_{2}=A_{n 2} \leq A_{n-1,2} \leq \cdots \leq$ $A_{12}$, we also have $n A_{2} \lesssim A_{12} \oplus A_{22} \oplus \cdots \oplus A_{n 2} \cong C_{11} \oplus C_{21} \oplus \cdots \oplus C_{n 1}=$ $C_{1}$.

Finally, wo apply the above procedure to the isomorphism $B_{2} \oplus C_{2} \cong$ $A_{2} \oplus C_{2}$. We obtain decompositions $B_{2}=B_{3} \oplus B_{4}$ and $A_{2}=A_{3} \oplus A_{4}$ and $C_{2}=C_{3} \oplus C_{4}$ such that $B_{3} \cong A_{3}$ and $B_{4} \oplus C_{4} \cong A_{4} \oplus C_{4}$, while $n B_{4} \lesssim C_{3}$. Set $A^{\prime}=A_{1} \oplus A_{3}$ and $A^{\prime \prime}=A_{4}$, and define $B^{\prime}, B^{\prime \prime}, C^{\prime}, C^{\prime \prime}$ 
similarly. Then $A=A^{\prime} \oplus A^{\prime \prime}$ and $B=B^{\prime} \oplus B^{\prime \prime}$ and $C=C^{\prime} \oplus C^{\prime \prime}$; with $A^{\prime} \cong B^{\prime}$ and $A^{\prime \prime} \oplus C^{\prime \prime} \cong B^{\prime \prime} \ominus C^{\prime \prime}$, while also $n\left(A^{\prime \prime} \oplus B^{\prime \prime}\right) \lesssim n A_{2} \oplus n B_{4} \lesssim$ $C_{1} \oplus C_{3}=C^{\prime}$.

Now we can give a different proof of $[\mathrm{O}$, Theorem 1$]$.

Theorem 1.11. (O'Meara) Let $R$ be a directly finile simple regulor ring satisfying weak comparability. Then $R$ is unit-regular.

Proof: We show that $R$ satisfies condition (b) in Theorem 1.9. Let $B, C \in L\left(R_{R}\right)$ with $R \oplus B \leq R \oplus C$. Given $0 \neq D \in L\left(R_{R}\right)$ thcre exists, by weak comparability, a positive integer $n$ such that $n T \lesssim R$ implies $T \lesssim D$ for any $T \in L\left(R_{R}\right)$.

By Lemma 1.10 there exists a decomposition $B=B^{\prime} \oplus B^{\prime \prime}$ such that $B^{\prime} \lesssim C$ and $n B^{\prime \prime} \lesssim R$. Consequently $B^{\prime \prime} \lesssim D$ and $B \lesssim C \oplus D$. It, follows that $B \lesssim_{a} C$ and thus $R$ is unit-regular by Theorem 1.9 .

\section{The almost isomorphism relation.}

Let $R$ be a non-artinian stably finite simple regular ring. $\mathrm{By}[\mathrm{B3}$, Theorem 3.1.4], the relation $\lesssim_{a \alpha}$ is cancellative, i.e. $A \oplus B \lesssim_{\alpha a} A \oplus C$ implies $B \lesssim_{a a} C$. So the approximately almost isomorphism classes of finitely generated projective modules form a cancellative abelian semigroup $S$ (since it is easy to show that direct sum gives a well-defined operation). Denote by $[A]_{a}$ the class of $A$ in $S$. We define a partial order on $S$ by $[A]_{a} \leq[B]_{a}$ if and only if $A \leqslant_{a a} B$. It is easy to show that this relation is well-defined and translation-invariant, so that $S$ becomes a partially ordered abelian semigroup. Also, the relation $\leq$ is cancellative, i.e. $x+y \leq z+y$ implics $x \leq z$ for $x, y, z \in S$, again by [B3, Theorem $3.1,4$. Let $K_{0}^{a}(R)$ be the abelian group obtained by adjoining inverses formally to $S$. Because of the cancellation property of $\leq$, the relation $x-y \leq z-t$ iff $x+t \leq z+y$ for $x, y, z, t \in S$ becomes a partial order in $K_{0}^{a}(R)$. It is easy to show that this partial order is translation-invariant and so $K_{0}^{\alpha}(R)$ becomes a partially ordered abelian group, in which we fix the order-unit $[R]_{a}$.

Proposition 2.1. Let $R$ be a non-artinian stably finite simple regular ring. Let $\Phi: K_{0}(R) \longrightarrow \operatorname{Aff}\left(S\left(K_{0}(R),[R]\right)\right)$ be the natural map. Assume that $\Phi\left(K_{0}(R)\right)$ is endowed with the partial order $f \leq g$ iff $f(x) \leq g(x)$ for all $x \in S\left(K_{0}(R),[R]\right)$. Then $\Phi\left(K_{0}(R)\right) \cong K_{0}^{R}(R)$ as partially ordered abelian groups with order-unit.

Proof: We have a surjective positive homomorphism $\alpha: K_{0}(R) \longrightarrow$ $K_{0}^{a}(R)$ given by $\alpha([A]-[B])=[A]_{a}-[B]_{\alpha}$. We will show that $\operatorname{Ker}(\alpha)=$ 
$\operatorname{Ker}(\Phi)$. It is clear that $\Phi([A]-[B])=0$ whenever $A \cong_{a a} B$, but this happens exactly when $[A]_{a}=[B]_{a}$. Conversely assume that $\Phi([A]-$ $[B])=0$. Let $0 \neq C \in L\left(R_{R}\right)$. Then $\Phi([A \oplus C]-[B]) \gg 0$ and so, by $[\mathbf{G 4}$, Theorem 4.12] there exists $m \geq 1$ such that $m([A \oplus C]-[B])>0$. By using [B3, Theorem 3.1.4] we have that there exists $n \geq 1$ such that $n m B \lesssim n m(A \oplus C)$. Consequently $B \leqslant_{a a} A$. Analogously $A \lesssim_{a a} B$ and so $[A]_{a}-[B]_{a}=0$.

It follows that we have a group isomorphism $\gamma: K_{0}^{a}(R) \rightarrow \Phi\left(K_{0}(R)\right)$ given by $\gamma\left([A]_{a}-[B]_{a}\right)=\Phi([A]-[B])$. Since $R$ is not artinian $\gamma$ is positive. Conversely, if $\Phi([A]-[B]) \geq 0$ then by the same argument as before we obtain that $B \lesssim_{a a} A$ and consequently $[A]_{a}-\{B]_{a} \geq 0$.

Henceforth, we will identify $K_{0}^{a}(R)$ with $\Phi\left(K_{0}(R)\right)$. The proof of the following lemma is straightforward.

Lemma 2.2. Let $R$ be a stably finite simple regular ring.

(a) Let $S=\lim _{\longrightarrow} M_{n}(R)$. Then $K_{0}(S)=K_{0}(R) \otimes \mathbf{Q}$.

(b) Let $S_{m}=\underline{\lim } M_{m^{k}}(R)$, for a fixed $m \geq 1$. Then $K_{0}\left(S_{m}\right)=$ $K_{0}(R) \otimes \mathbf{D}_{m}$ where $\mathbf{D}_{m}=\left\{a / m^{k} \mid a \in \mathbf{Z}, k \geq 1\right\}$.

By [B3, Theorem 3.1.4], the rings $S=\lim _{\rightarrow} M_{n}(R)$ and all $S_{n}=$ $\lim M_{m^{k}}(R)$ are unperforated unit-regular simple rings provided $R$ is $\vec{a}$ atably finite simple regular ring. It follows that $K_{0}(S)$ and all $K_{0}\left(S_{m}\right)$ are simple dimension groups. Therefore we can use [G4, Theorem 14.14] and Lemma 2.2 to study the question of when $\Phi\left(K_{0}(R)^{+}\right)$is dense in $\operatorname{Aff}\left(S\left(K_{0}(R),[R]\right)\right)^{+}$, leading to the following lemma.

Recall that for any partially ordered abelian group $G$ and any subgroup $H$ of $\mathrm{Q}$, the tensor product $G \otimes H$ is a partially ordered abelian group with positive cone $(G \otimes H)^{+}=\left\{x \otimes y \mid x \in G^{+}, y \in H^{+}\right\}$.

Lemma 2.3. Let $H=\mathbf{Q}$ (resp. $H=\mathbf{D}_{m}$ ), endowed with the usual order. Let $(G, u)$ be a partially ordered simple abelian group with orderunit, and assume that $G \otimes H$ is a simple dimension group. Let $\Phi$ : $G \longrightarrow \operatorname{Aff}(S(G, u))$ be the natural map. Then the following properties are equivalent:

(a) $\Phi\left(G^{+}\right)$is dense in $\operatorname{Aff}(S(G, u))^{+}$.

(b) For each $0 \neq x \in G^{+}$, for each $n \geq 1$ (resp. for each $n=m^{k}$, with $k \geq 0$ ), and for each $\epsilon>0$ there exist $y_{1}, y_{2} \in G^{+}$such that

$$
\begin{aligned}
& n \Phi\left(y_{2}\right) \ll \Phi(x) \ll n \Phi\left(y_{1}\right) \\
& n \Phi\left(y_{1}\right)-\Phi(x) \ll \epsilon \\
& \Phi(x)-n \Phi\left(y_{2}\right) \ll \epsilon
\end{aligned}
$$


(c) For each $0 \neq x \in G^{+}$, for each $n \geq 1$ (resp. for each $n=m^{k}$, with $k \geq 0$ ), and for each $\epsilon>0$ there exists $y_{1} \in G^{+}$such that $\Phi(x) \ll n \Phi\left(y_{1}\right)$ and $n \Phi\left(y_{1}\right)-\Phi(x) \ll \epsilon$.

Proof:

(a) $\Rightarrow$ (b):

Assume that $\Phi\left(G^{+}\right)$is dense in $\operatorname{Aff}(S(G, u))^{+}$. Now fix $0 \neq x \in$ $G^{+}, n \geq 1$ and $\epsilon>0$. Since $G$ is simple, $\Phi(x) \gg 0$.

Hence, after possibly replacing $\epsilon$ by a smaller positive real number, we may assume that $\Phi(x) \gg \frac{\epsilon}{2}$. Thus the functions $f_{1}=\frac{1}{n} \Phi(x)+\frac{\epsilon}{2 n}$ and $f_{2}=\frac{1}{n} \Phi(x)-\frac{c}{2 n}$ are positive. Since $\Phi\left(G^{+}\right)$is dense in $\operatorname{Aff}(S(G, u))^{+}$, there cxist $y_{1}, y_{2} \in G^{+}$such that $\left\|\Phi\left(y_{i}\right)-f_{i}\right\|<\frac{\epsilon}{2 n}$ for $i=1$, 2. Then

$$
\begin{aligned}
& \Phi(x)=n f_{1}-\frac{\epsilon}{2} \ll n \Phi\left(y_{1}\right) \ll n f_{1}+\frac{\epsilon}{2}=\Phi(x)+\epsilon \\
& \Phi(x)-\epsilon=n f_{2}-\frac{\epsilon}{2} \ll n \Phi\left(y_{2}\right) \ll n f_{2}+\frac{\epsilon}{2}=\Phi(x) .
\end{aligned}
$$

So, $y_{1}, y_{2} \in G^{+}$satisfy the required conditions.

Obviously, (b) $\Rightarrow$ (c).

(c) $\Rightarrow(\mathrm{a})$ :

Let $\tau: G \longrightarrow G \otimes H$ be the map given by $\tau(x)=x \otimes 1 . \tau$ induces $\tau_{*}: S(G \otimes H, u \otimes 1) \longrightarrow S(G, u)$. If $s=\tau_{*}\left(s^{\prime}\right)$ with $s^{\prime} \in S(G \otimes H, u \otimes 1)$ then $s^{\prime}(x \otimes 1 / n)=\frac{1}{n} s(x)$ for $\frac{1}{n} \in H$. This implies that $\tau_{*}$ is injective. On the other hand, if $s \in S(G, u)$ then the expression $s^{\prime}\left(x \otimes \frac{1}{n}\right)=\frac{1}{n} s(x)$ defines $s^{\prime} \in S(G \otimes H, u \otimes 1)$ such that $\tau_{*}\left(s^{\prime}\right)=s$. It follows that $\tau_{*}$ is an affine homeomorphism. Now we have the following commutative diagram:

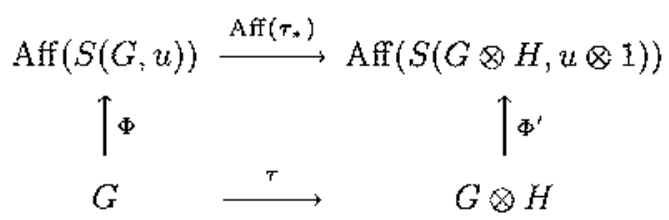

and Aff $\left(\tau_{*}\right)$ is an isomorphism of partially ordered abelian groups with order-unit. Since $G \otimes H$ is a simple dimension group, $\Phi^{\prime}\left((G \otimes H)^{+}\right)$is dense in $\operatorname{Aff}(S(G \otimes H, u \otimes 1))^{-}$by [G4, Theorem 14.14]. So $\Phi\left(G^{+}\right)$is dense in $\operatorname{Aff}(S(G, u))^{+}$if and only if $\operatorname{Aff}\left(\tau_{*}\right)\left(\Phi\left(G^{+}\right)\right)$is dense in $\Phi^{\prime}((G \otimes$ $H)^{+}$).

Let $0 \neq \Phi^{\prime}(x \otimes 1 / n)$ and $\epsilon>0$, with $x \in G^{+}$and $1 / n \in H$. Then there exists $y_{1} \in G^{+}$with $\Phi(x) \ll n \Phi\left(y_{1}\right)$ and $n \Phi\left(y_{1}\right)-\Phi(x) \ll \epsilon$. It follows that

$$
\left\|\operatorname{Aff}\left(\tau_{*}\right)\left(\Phi\left(y_{1}\right)\right)-\Phi^{\prime}\left(x \otimes \frac{1}{n}\right)\right\|<\frac{\epsilon}{n} \leq \epsilon
$$


and consequently $\Phi\left(G^{+}\right)$is dense in $\operatorname{Aff}(S(G, u))^{+}$.

For a stably finite simple regular ring $R$, the condition $\Phi([A]) \ll$ $\Phi([B])$ is equivalent to $t A \prec t B$ for some $t \geq 1$, by [G4, Theorem 4.12] and [B3, Theorem 3.1.4]. By using this and Lemma 2.3, we obtain the following Proposition.

Proposition 2.4. Let $R$ be a stably finite simple regular ring. Then the following conditions are equivalent:

(1) $\Phi\left(K_{0}(R)^{+}\right)$is dense in Aff $\left(S\left(K_{0}(R),(R)\right)\right)^{+}$.

(2) For any $A \in \mathcal{P}_{0}, n \geq 1$ and $\epsilon>0$ there exist $B, C \in \mathcal{P}$ such that $t A \prec n t B$ for some $t \geq 1$ while $\left.n[B]_{a}-\mid A\right]_{a} \ll \epsilon$; and nr $C \prec r A$ for some $r \geq 1$ while $[A]_{a}-n[C]_{a} \ll \epsilon$.

(3) There exists $m \geq 2$ such that for all $A \in \mathcal{P}, k \geq 1$ and $c>0$, there exists $B \in \mathcal{P}$ such that $t A \prec m^{k} t B$ for some $t \geq 1$ while $m^{k}[B]_{a}-[A]_{a} \ll \epsilon$.

We will call condition (I) in the above Proposition condition (D).

Corollary 2.5. If $R$ is a stably finite simple regular ring and there exists $m \geq 2$ such that eRe is an $m \times m$ matrix ring for each idempotent $e \in R$, then $R$ satisfies condition (D).

Corollary 2.6. Let $R$ be a stably finite simple regular ring satisfying condition $(D)$. Then $\left(K_{0}^{a}(R), \ll\right)$ is a simple dimension group.

Proof: We observe that $\Phi\left(K_{0}(R)^{+}\right) \subseteq K_{0}^{a}(R)^{+}$. Since $R$ satisfics (D), $K_{0}^{a}(R)^{+}$is dense in Aff $\left(S\left(K_{0}(R),[R]\right)\right)^{+}$. Consequently $K_{0}^{a}(R)$ is dense in $\operatorname{Aff}\left(S\left(K_{0}(R),[R]\right)\right)$ and thus by [G4, Prop. 14.15] $\left(K_{0}^{a}(R), \ll\right)$ is a simple dimension group.

The following corollary is a consequence of the fact that $\left(K_{0}^{a}(R), \ll\right)$ is an interpolation group whenever $R$ satisfies condition (D).

Corollary 2.7. Let $R$ be a stably finite simple regular ring satisfying condition (D). If $n A \prec n(B \oplus C)$ for $A, B, C \in \mathcal{P}_{0}$, then for each $\epsilon>0$ there exists $A_{1}, A_{2}, A_{1}^{\prime}, A_{2}^{\prime} \in \mathcal{P}$ and $m \geq 1$ such that $m\left(A_{1}^{\prime} \in A_{2}^{\prime}\right) \prec$ $m A \prec m\left(A_{1} \oplus A_{2}\right)$, and $m A_{1}^{\prime} \prec m A_{1} \prec m B$ and $m A_{2}^{\prime} \prec m A_{2} \prec m C$, while also $\left[A_{1}\right]_{a}+\left[A_{2}\right]_{a}-[A]_{a} \ll \epsilon$ and $[A]_{a}-\left[A_{1}^{\prime}\right]_{a}-\left[A_{2}^{\prime}\right]_{a} \ll \epsilon$.

Proof: If $n A \prec n(B \oplus C)$, then we have $[A]_{a} \ll[B]_{a}+[C]_{a}$. Since $\left(K_{0}^{a}(R), \ll\right)$ is an interpolation group by Corollary 2.6 , there exist $X_{1}^{t}$ : $X_{2}^{\prime} \in K_{0}^{a}(R)^{++}$such that $[A]_{a}=X_{1}^{\prime}+X_{2}^{\prime}$ while $X_{1}^{\prime} \ll[B]_{a}$ and $X_{2}^{\prime} \ll[C]_{0}$. Clearly, one of the incqualities must be strict, so assume that $X_{1}^{\prime} \ll[B]_{a}$. 
If also $X_{2}^{\prime} \ll[C]_{a}$, put $X_{1}=X_{1}^{\prime}, X_{2}=X_{2}^{\prime}$. If $X_{2}^{\prime}=[C]_{a}$ then write $X_{1}^{\prime}=\left[Z_{1}\right]_{a}-\left[Z_{2}\right]_{a}$ for some $Z_{1}, Z_{2} \in \mathcal{P}$, and observe that there exists $k \geq 1$ such that $k Z_{2} \prec k Z_{1}$ and $k Z_{1} \prec k\left(B \oplus Z_{2}\right)$. Choose $Z \in \mathcal{P}_{0}$ such that $Z \prec C$ and $k\left(Z_{1} \oplus Z\right) \prec k\left(B \oplus Z_{2}\right)$. Set $X_{1}=X_{1}^{\prime}+[Z]_{a}$ and $X_{2}=$ $X_{2}^{\prime}-[Z]_{a}$ and observe that $X_{1}, X_{2} \in K_{0}^{a}(R)^{++}$satisfy $[A]_{a}=X_{1}+X_{2}$, and $X_{1} \ll[B]_{a}$ and $X_{2} \ll[C]_{a}$.

Now write $X_{1}=\left[T_{1}\right]_{a}-\left[T_{2}\right]_{a}$ for some $T_{1}, T_{2} \in \mathcal{P}$. Then we have $k T_{2} \prec k T_{1}$ for some $k \geq 1$ and so we can write $k T_{1} \cong k T_{2} \oplus S$ for some $S \in \mathcal{P}_{0}$. Observe that $X_{1}=\frac{1}{k}[S]_{a}$. Choose $\epsilon_{1}>0$ such that $\epsilon_{1} \ll[B]_{a}-X_{1}$ and $\epsilon_{1} \leq \epsilon / 2$. By Proposition 2.4 there exist $A_{1}^{\prime}, A_{1} \in \mathcal{P}$ such that $k t A_{1}^{\prime} \prec t S \prec k t A_{1}$ for some $t \geq 1$ while also $k\left[A_{1}\right]_{a}-[S]_{a} \ll \epsilon_{1}$ and $[S]_{a}-k\left[\dot{A}_{1}^{\prime}\right]_{a} \ll \epsilon_{1}$.

We have $\left[A_{1}^{\prime}\right]_{a} \ll X_{1} \ll\left[A_{1}\right]_{a}$ and also $\left[A_{1}\right]_{a}-X_{1} \ll \epsilon_{1} / k \leq \epsilon_{1} \leq \epsilon / 2$ and $X_{1}-\left[A_{1}^{\prime}\right]_{a} \ll \epsilon_{1} / k \leq \epsilon_{1} \leq \epsilon / 2$. It follows that $[B]_{a} \gg X_{1}+\epsilon_{1} \gg$ $\left[A_{1}\right]_{a}$. Similarly there exist $A_{2}, A_{2}^{\prime} \in \mathcal{P}$ such that $\left[A_{2}^{\prime}\right]_{a} \ll X_{2} \ll\left[A_{2}\right]_{a}$ with $\left[A_{2}\right]_{a}-X_{2} \ll \epsilon / 2$ and $X_{2}-\left[A_{2}^{\prime}\right]_{a} \ll \epsilon / 2$, while also $[C]_{a} \gg\left[A_{2}\right]_{a}$. Consequently $\left[A_{1}^{\prime} \oplus A_{2}^{\prime}\right]_{a} \ll X_{1}+X_{2}=[A]_{a} \ll\left[A_{1} \oplus A_{2}\right]_{a}$ and also $[A]_{a}-\left[A_{1}^{\prime}\right]_{a}-\left[A_{2}^{\prime}\right]_{a} \ll \epsilon$ and $\left[A_{1}\right]_{a}+\left[A_{2}\right]_{a}-[A]_{a} \ll \epsilon$, as required.

We say that $R$ is strictly m-unperforated if $m A \prec m B$ implies $A \prec$ $B$ for all $A, B \in \mathcal{P}$. So, $R$ is strictly unperforated if it is strictly $m$ unperforated for all $m \geq 1$.

Corollary 2.8. Let $R$ be a non-artinian directly finite simple regular ring which is strictly $m$-unperforated for some $m \geq 2$. Then $R$ is unitregular, strictly unperforated, satisfies property $(D)$ and, moreover, for any $A \in \mathcal{P}_{0}, n \geq 1$ and $\epsilon>0$, the following conditions hold:

(a) If $B \in \mathcal{P}$ and $B \prec A$, then there exists $T \in \mathcal{P}$ such that $B \prec$ $n T \prec A$ and $[A]_{a}-n[T]_{a} \ll \epsilon$.

(b) If $D \in \mathcal{P}$ and $A \prec D$, then there exists $V \in \mathcal{P}$ such that $A \prec$ $n V \prec D$ and $n[V]_{a}-[A]_{a} \ll \epsilon$.

Proof: $R$ is unit-regular by [O, Theorem 1].

$R$ is strictly unperforated by [B3, Theorem 2.1.11]. By Proposition A1, $R$ satisfies property (D).

Since $R$ is strictly unperforated, (a) and (b) follows from Proposition 2.4 by using the same arguments as in Corollary 2.7 .

The following observation is a consequence of Corollary 2.8 .

Remark 2.9. Let $R$ be a directly finite simple regular ring which is strictly $m$-unperforated for some $m \geq 2$. Then $A \lesssim_{a a} B$ if and only if $A \Sigma_{2} B$, for $A, B \in \mathcal{P}$. 


\section{The doubling condition.}

We will say that a stably finite regular ring $R$ satisfies the doubling condition (DD) if for every nonzero $D \in L\left(R_{R}\right)$ there exists $K \geq 1$ such that, for $A \in L\left(R_{R}\right)$, if $A \leq D$ and $K[A]_{a} \ll[D]_{a}$ then $2 A \leqq D$.

Lemma 3.1. Let $R$ be a stably finite simple regular ring. Then $R$ satisfies (DD) if and only if for every $D \in \mathcal{P}$ there exists $K \geq 1$ such that, for $A \in \mathcal{P}$, if $A \leq D$ and $K[A]_{a} \ll[D]_{a}$ then $2 A \leq D$.

Proof: Assume that $R$ satisfies (DD). Write $D=D_{1} \oplus \cdots \odot D_{r}$, where $0 \neq D_{i} \lesssim R$. For $i=1, \ldots, r$, there exist $K_{i} \geq 1$ such that, for $A_{i} \in$ $L\left(R_{R}\right)$, if $A_{i} \lesssim D_{i}$ and $K_{i}\left[A_{i}\right]_{a} \ll\left[D_{i}\right]_{a}$ then $2 A_{i} \lesssim D_{i}$. Take:

$$
K \geq \max \left\{K_{\imath}[D]_{a} /\left[D_{i}\right]_{a} \mid i=1, \ldots, r\right\} .
$$

Lemma 3.2. Let $R$ be a direclly finite simple regular ring such that $n(x R) \prec n(y R)$ implies $x R \prec y R$ for all $x, y \in R$. Then $R$ satisfies the doubling condition with $K=2$.

Proof: It follows from $[\mathrm{O}$, Theorem 1] that $R$ is unit-regular. Put $D=y R$ and $A=x R$, and assume that $A \leq D$. Write $D=A \oplus T$. Assume that $2[A]_{a} \ll[D]_{a}$. Then $2 n A \prec n D \cong n A \oplus n T$ for some $n \geq 1$. Since $R$ is unit-regular $n A \prec n T$. By hypothesis $A \prec T$. So we obtain property (DD) with $K=2$.

Lemma 3.3. Let $R$ be a regular ring and $B, D \in \mathcal{P}$ with $B \leqslant r D$ for some $r \geq 1$. Then there exists a decomposition $B=B_{1} \oplus \cdots \oplus B_{r}$. such that $B_{1} \lesssim B_{2} \lesssim \cdots \leqq B_{r} \lesssim D$.

Proof: In case $r=1$, there is nothing to prove. Now assume that $r>1$, and choose a decomposition $B=B_{1}^{\prime} \ominus \cdots \ominus B_{r}^{\prime}$ such that, $B_{i}^{\prime} \leqq D$ for all $i$. Choose $D_{i}^{\prime} \leq D$ such that $B_{i}^{\prime} \cong D_{i}^{\prime}$, and set $D^{\prime}=D_{1}^{\prime}+\cdots+D_{r}^{\prime} \leq$ $D$. Then there exists an epimorphism $\phi: B \longrightarrow D^{\prime}$ induced by the isomorphisms from $B_{i}^{\prime}$ onto $D_{i}^{\prime}$. Since $B_{r}^{\prime} \cap \mathrm{Kcr}(\phi)=0$, we must have $B=B_{r}^{\prime} \odot \operatorname{Ker}(\phi) \odot T$ for some $T$. Note that $B_{r}:=B_{r}^{\prime} \oplus T$ is isomorphic to $D^{\prime}$. Also, $T^{\prime}:=\operatorname{Ker}(\phi) \ominus T$ satisfies $T^{\prime} \ominus B_{r}^{\prime}=B=\left(B_{1}^{\prime} \odot \cdots \oplus B_{r-1}^{\prime}\right) \oplus B_{r}^{\prime}$ and so $T^{\prime} \cong B_{1}^{\prime} \ominus \cdots \Theta B_{r-1}^{\prime}$. Now $\operatorname{Ker}(\phi) \leq T^{\prime \prime} \leqq(r-1) D^{\prime} \cong(r-1) B_{r}$. By induction, there exists a decomposition $\operatorname{Ker}(\phi)=B_{1} \odot \cdots \oplus B_{r-1}$ such that $B_{1} \leqq B_{2} \lesssim \cdots \leqq B_{r-1} \lesssim B_{r}$. Thus $B=B_{1} \ominus \cdots \oplus B_{r-1} \odot B_{r}$ and the result follows.

We thank $E$. Pardo for a simplification of the original version of the following proposition. 
Proposition 3.4. Let $R$ be a stably finite simple regular ring. Then the following conditions are equivalent:

(a) $R$ satisfies $(D D)$.

(b) For any $D \in L\left(R_{R}\right)$ there exists $K \geq 1$ such that, for $A \in L\left(R_{R}\right)$, if $K[A]_{a} \ll[D]_{a}$ then $A \prec D$.

(c) For any $D \in \mathcal{P}$ there exists $K \geq 1$ such that, for $A \in \mathcal{P}$, if $K[A]_{a} \ll[D]_{a}$ then $A \prec D$.

Proof: Obviously (c) $\Rightarrow$ (b).

(b) $\Rightarrow$ (a): Clearly, we can suppose that $R$ is not artinian. Let $D \in$ $L\left(R_{R}\right)$ and write $D=E_{1} \oplus E_{2} \oplus E_{3}$, where $E_{1} \neq 0$ and $E_{2} \cong E_{1}$. There exists $K^{\prime} \geq 1$ such that, for $B \in L\left(R_{R}\right)$, if $K^{\prime}[B]_{a} \ll\left[E_{1}\right]_{a}$ then $B \prec E_{1}$. Choose a constant $K \geq K^{\prime}[D]_{a} /\left[E_{1}\right]_{\alpha}$. Then it is clear that, for $A \in L\left(R_{R}\right)$, if $K[A]_{a} \ll[D]_{a}$ then $2 A \prec E_{1} \oplus E_{2} \leq D$.

(a) $\Rightarrow$ (c): We may suppose that $D \neq 0$. Choose a nonzero $E \in L\left(R_{R}\right)$ such that $E \leqq D$. By simplicity, $D \leqq m E$ for some $m \geq 1$. By (DD), there exists $K^{\prime} \geq 1$ such that for $A \in L\left(R_{R}\right)$, if $A \leq E$ and $K^{\prime}[A]_{a} \ll$ $[E]_{a}$ then $2 A \lesssim E$.

Set $K=K^{\prime} m$, and consider $A \in \mathcal{P}$ such that $K[A]_{a} \ll[D]_{a}$; then $K^{\prime}[A]_{a} \ll[E]_{a}$. By simplicity, $A \lesssim r E$ for some $r \geq 1$. Hence, by Lemma 3.3 there exists a decomposition $A=A_{1} \oplus \cdots \oplus A_{r}$ such that $A_{1} \lesssim A_{2} \lesssim \cdots \lesssim A_{r} \lesssim E$. Since $A_{r} \lesssim E$ and $K^{\prime}\left[A_{r}\right]_{a} \leq K^{\prime}[A]_{a} \ll[E]_{a}$, we obtain $2 A_{r} \lesssim E$. Now $A_{r-1} \oplus A_{r} \lesssim 2 A_{r} \lesssim E$ and $K^{\prime}\left[A_{r+1} \oplus A_{r}\right]_{a} \leq$ $K^{\prime}[A]_{a} \ll[E]_{a}$, whence $2\left(A_{r-1} \oplus A_{r}\right) \lesssim E$. Continuing by induction, we find that $2 A \lesssim E$, and therefore $A \prec D$.

Let $R$ be a directly finite simple regular ring. We say that $R$ satisfies (dd) if for any $D \in L\left(R_{R}\right)$ there exists $n \geq 1$ such that, for $A \in L\left(R_{R}\right)$, if $A \leq D$ and $n A \lesssim R$ then $2 A \lesssim R$. The proof of the following proposition is analogous to that of Proposition 3.4 , so that we will omit it.

Proposition 3.5. Let $R$ be a directly finite simple regular ring. Then $R$ satisfies weak comparability if and only if $R$ satisfies (dd).

Corollary 3.6. Let $R$ be a stably finite simple regular ring satisfying $(D D)$. Then $M_{n}(R)$ satisfies weak comparability for all $n \geq 1$, In particular, $R$ is unit-regular.

Proof: By Lemma 3.1, $M_{n}(R)$ satisfy (DD) for all $n \geq 1$. Since property (DD) obviously implies property (dd), the result follows from Proposition 3.5 and Theorem 1.11 . 


\section{Simple regular rings with a unique rank function.}

Let $R$ be a regular ring and let $s, t$ be positive integers. Consider the following comparability conditions:

aa-comparability: For $x, y \in R$, either $x R \S_{a \alpha} y R$ or $y R \lesssim_{a a} x R$.

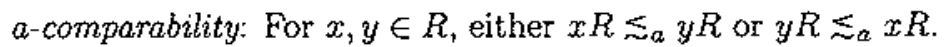

(s:t)-comparability $([G 1, p .275])$ : For any $x, y \in R$, either $t(x R) \lesssim$ $s(y R)$ or $t(y R) \leq s(x R)$.

approximate (s:t)-comparability $([\mathbf{G} 1$, p. 275]): For any $x, y \in R$, there exists a positive integer $n$ such that either $n t(x R) \lesssim n s(y R)$ or $n t(y R) \lesssim$ $n s(x R)$.

In case $t=1$ we abbreviate the two latter terms to "s-comparability" and "approximate s-comparability" respectively.

Proposition 4.1. (cf. /G1, Theorem 18.17]) Let $R$ be a stably finite simple regular ring. Then the following conditions are equivalent:

(a) $R$ has a unique rank function.

(b) $R$ satisfies the aa-comparability condition.

(c) $R$ satisfies approximate (s.t)-comparability for all integers $0<t<$ $s$.

(d) $R$ satisfies approximate (s:t)-comparability for some integers $s \geq$ $t>0$.

Proof: We can assume that $R$ is not artinian.

By [G1, Theorem 18.3], $R$ has a rank function $N$.

(a) $\Rightarrow$ (b): Assume that $N$ is the unique rank function on $R$. Then, given $x, y \in R$ either $[x R]_{a}-[y R]_{a}>0,[y R]_{a}-[x R]_{a}>0$ ог $[x R]_{a}=$ $[y R]_{2}$. This corresponds to $n(y R) \prec n(x R)$ some $n, m(x R) \prec m(y R)$ some $m$, or $x R \cong_{a a} y R$, respectively. Thus, in any case, either $x R \lesssim_{a a}$ $y R$ or $y R \Xi_{a a} x R$.

(b) $\Rightarrow$ (c): Let $s$ and $t$ be integers such that $0<t<s$ and let $0 \neq x, y \in R$. Assume that $x R \lesssim_{a a} y R$. Choose $0 \neq z \in R$ such that $t(z R) \leqq y R$. There exists $n \geq I$ such that $n(x R) \lesssim n(y R \oplus z R)$ and so

$$
n t(x R) \lesssim n t(y R) \oplus n t(z R) \lesssim n s(y R) .
$$

Consequently, $R$ satisfies approximate (s:t)-comparability.

(c) $\Rightarrow$ (d): Obvious

(d) $\Rightarrow$ (a): The same proof as in [G1, Theorem 18.17] applies.

Corollary 4.2. Let $R$ be a stably finite simple regular ring. If $R$ satisfies the aa-comparability condition then so does $M_{n}(R)$ for all $n \geq$ 
1. Moreover, given finitely generated projective modules $A, B$, either $n A \prec n B$ for some $n \geq 1, m B \prec m A$, for some $m \geq 1$, or $A \cong_{a a} B$

Proof: Since $M_{n}(R)$ has a unique rank function the above proof applics to finitely generated projective modules.

Proposition 4.3. Let $R$ be a directly finite simple regular ring satisfying a-comparability. Then $R$ is unit-regular.

Proof: It is clear that $R$ satisfics 2-comparability. By $[\mathrm{O}$, Corollary 2], $R$ is unit-regular.

By a slight modification of the proof in [G1, Proposition 8.2], we obtain the following result.

Proposition 4.4. Let $R$ be a directly finite simple regular ring. If $R$ satisfies the a-comparability condition, then so does $M_{n}(R)$ for all $n \geq 1$.

Proof: We can assume that $R$ is not artinian.

We will prove that for finitely gencrated projective modules $A, B$, either $A \varsigma_{a} B$ or $B \varsigma_{a} A$. By induction, assume the result is true for proper submodules of $(n-1) R$, and let $A, B$ with $A, B<n R$.

Write $A=A_{1} \oplus A_{2}, B=B_{1} \oplus B_{2}$ with $A_{1}, A_{2}, B_{1}, B_{2} \prec(n-1) R$. Now either $A_{1} \lesssim_{a} B_{1}$ or $B_{1} \lesssim_{0} A_{1}$, and either $A_{2} \lesssim_{a} B_{2}$ or $B_{2} \Xi_{a} A_{2}$. We need only consider the case where $A_{1} \lesssim_{a} B_{1}$ and $B_{2} \Xi_{a} A_{2}$. Let $0 \neq C \in$ $L\left(R_{R}\right)$ be such that $B_{1} \ominus C \prec(n-1) R$ and $\Lambda_{2} \ominus C \prec(n-1) R$. Then $A_{1} \leqq B_{1} \oplus C$ and $B_{2} \lesssim A_{2} \oplus C$, so $B_{1} \ominus C=B_{1}^{\prime} \oplus B_{1}^{\prime \prime}, A_{2} \oplus C=A_{2}^{\prime} \oplus A_{2}^{\prime \prime}$ with $B_{1}^{\prime} \cong A_{1}$ and $A_{2}^{\prime} \cong B_{2}$. So cither $B_{1}^{\prime \prime} \Xi_{a} A_{2}^{\prime \prime}$ or $A_{2}^{\prime \prime} \Xi_{0} B_{1}^{\prime \prime}$. Assume that $B_{1}^{\prime \prime} \S_{a} A_{2}^{\prime \prime}$. Then $B_{1} \oplus B_{2} \oplus C \cong B_{1}^{\prime} \oplus B_{1}^{\prime \prime} \oplus A_{2}^{\prime} \lesssim_{a} B_{1}^{\prime} \oplus A_{2}^{\prime \prime} \oplus A_{2}^{\prime} \cong$ $A_{1} \oplus A_{2} \ominus C$, and so $B_{1} \oplus B_{2} \lesssim_{a} A_{1} \oplus A_{2}$, since $R$ is unit-regular by Proposition 4.3.

Proposition 4.5. Let $R$ be a simple regular ring with a unique rank function $N$. Then the following are equivalent:

(a) $R$ satisfies the a-comparability oxiom.

(b) $R$ is strictly unperforated.

(c) For $x, y \in R, n(x R) \prec n(y R)$ implies $x R \prec y R$

Proof: We can assume that $R$ is not artinian.

(a) $\Rightarrow$ (b): Assume that, $n A \prec n B$. If $B \lesssim_{a} A$ then write $n B=T_{1} \odot T_{2}$ with $T_{1} \cong n A$ and $T_{2} \neq 0$. Choose $0 \neq T$ with $n T \prec T_{2}$. Then $B \lesssim A \oplus T$ so $n B \leqq n A \oplus n T \prec T_{1} \oplus T_{2}=n B$, contradiction. So $A \lesssim_{a} B$. Applying 
the same argument to $A \oplus T$, we see that $A \oplus T \lesssim_{\alpha} B$. Consequently, $A \oplus T \prec B \oplus T$ and so, $A \prec B$ because $R$ is unit-regular.

(b) $\Rightarrow$ (c): Obvious.

(c) $\Rightarrow$ (a): Since $R$ has a unique rank function, we see from Proposition 4.1 that either $x R \lesssim_{a a} y R$ or $y R \lesssim_{\alpha a} x R$. If $x R \lesssim_{a \alpha} y R$, then for $0 \neq C \in L\left(R_{R}\right)$ we have $n(x R) \prec n(y R \oplus C)$, and so $x R \prec y R \oplus C$ provided that $y R \oplus C \lesssim R$. We can always assume this except in the case where $y R=R$. But if $y R=R$ then $x R \Sigma_{a} R=y R$ obviously. Consequently, $R$ satisfies a-comparability.

\section{Appendix.}

We prove the following result:

Proposition A1. Let $R$ be a strictly unperforated non-artinion simple unit-regular ring. Let $\Phi: K_{0}(R) \longrightarrow \operatorname{Aff}\left(S\left(K_{0}(R),[R]\right)\right)$ be the natural map. Then $\Phi\left(K_{0}(R)^{+}\right)$is dense in $\operatorname{Aff}\left(S\left(K_{0}(R),[R]\right)\right)^{+}$.

To prove Proposition A1, it clearly suffices to prove a corresponding result for partially ordered abelian groups (Theorem A3).

Note that if $G$ is a partially ordered abelian group then its torsion subgroup $T$ is a convex subgroup, and so $G / T$ is a partially ordered abelian group with respect to the induced ordering. A special case of a result of Elliott [E, Theorem 4.5] says that if $G$ is a strictly unperforated interpolation group, then $G / T$ is an unperforated interpolation group. Since the proof of this case is much easier than the proof of $[\mathrm{E}$, Theorem $4.5]$, we give the details.

Proposition A2. (Elliott) Let $G$ be a directed strictly unperforated interpolation group, and let $T$ be its torsion subgroup. Then $G / T$ is a dimension group.

Proof: It is clear that since $G$ is directed, so is $G / T$.

First consider $x \in G$ and $n \in \mathrm{N}$ such that $n(x+T) \geq 0$. If $x \in T$, then $x+T=0$, and so we may assume that $x \notin T$. Now $n x+T=y+T$ for some $y \in G^{+}$, and $y>0$ because $x \notin T$. Then $k(n x-y)=0$ for some $k \in \mathbb{N}$, whence $k n x=k y>0$. Since $G$ is strictly unperforated, $x>0$, and hence $x+T>0$. Thus $G / T$ is unperforated.

Now consider $x_{1}, x_{2}, y_{1}, y_{2} \in G$ such that $x_{i}+T \leq y_{j}+T$ for all $i, j$. If $x_{r}+T=y_{s}+T$ for some $r, s$, then $x_{i}+T \leq x_{r}+T \leq y_{j}+T$ for all $i, j$. Hence, we may assume that $x_{i}+T<y_{j}+T$ for all $i, j$. Consequently, there are nonzero elements $w_{i j} \in G^{+}$such that $x_{i}+w_{i j}+T=y_{j}+T$. There is some $k \in \mathbf{N}$ such that $k\left(x_{i}+w_{i j}-y_{j}\right)=0$ for all $i, j$, whence 
$k x_{i}<k y_{j}$ and so $x_{i}<y_{j}$ for all $i, j$, by strict unperforation. Thus there exists $z \in G$ such that $x_{i} \leq z \leq y_{j}$ for all $i, j$, and hence $x_{i}+T \leq z+T \leq$ $y_{3}+T$ for all $i, j$. Therefore $G / T$ is an interpolation group.

Theorem A3. Let $(G, u)$ be a strictly unperforated simple interpolation group with order-unit, such that $G^{+}$contains no atoms. Let $\Phi: G \longrightarrow \operatorname{Aff}(S)$ be the nalural map, where $S=S(G, u)$. Then $\Phi\left(G^{+}\right)$ is dense in $\mathrm{Aff}(S)^{+}$.

Proof: Let $T$ be the torsion subgroup of $G$, and note that $G / T$ is simple and that the element $u^{\prime}:=u+T$ is an order-unit in $G / T$. By Proposition A2, $G / T$ is a dimension group.

Suppose that $\left(G / T^{+}\right)^{+}$contains an atom, say $x+T$ where $x \in G^{+}$. Since $x$ cannot be an atom in $G^{+}$, there exists $y \in G$ such that $0<y<x$. But then $0+T<y+T<x+T$ (because $T^{\prime} \cap G^{+}=\{0\}$ ), contradicting our assumption about $x+T$. Therefore $(G / T)^{+}$contains no atoms.

Let $\pi: G \rightarrow G / T$ be the quotient map, and set $S^{t}=S\left(G / T, u^{\prime}\right)$. The induced map $\pi^{*}: S^{\prime} \rightarrow S$ is an affine homeomorphism, and hencc the induced map $\pi^{* *}: \operatorname{Aff}(S) \rightarrow \operatorname{Aff}\left(S^{\prime}\right)$ is an isomorphism of ordered Banach spaces. There is a commutative diagram as follows, where $\Phi^{\prime}$ is the natural map.

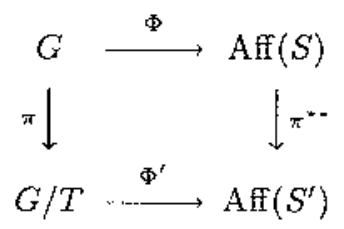

Since $(G / T)^{+}=\pi\left(G^{+}\right)$, it suffices to prove that $\Phi^{\prime}\left((G / T)^{+}\right)$is dense in $\operatorname{Aff}\left(S^{\prime}\right)^{+}$. Thus there is no loss of gencrality in assuming that $G$ is a simple dimension group, with no atoms in $G^{+}$.

Since $G^{+}$has no atoms, $G$ is not cyclic. Therefore; by [G4, Theorem 14.14], $\Phi\left(G^{+}\right)$is dense in $\operatorname{Aff}(S)^{+}$.

\section{Acknowledgement.}

It is a pleasure to thank J. Moncasi for his helpful comments.

Note added in proof. E. Pardo has proved that any non-artinian stably finite simple regular ring satisfies condition (D). 


\section{References}

[B1] B. BLACKADAR, A stable cancellation theorem for simple $C^{*}$-algebras, Proc. London Math. Soc. 47 (1983), 303-305.

[B2] B. Blackadar, Comparison theory for simple $C^{*}$-algebras, in "Operator Algebras and Application", D.E. Fvans and M. Takesaki (eds.), LMS Lecture Notes Series 135, Cambridge Univ. Press, 1988, pp. $21-54$.

[B3] B. BLACKADAR, Rational $C^{*}$-algebras and nonstable $K$-theory, Rocky Mountain J. Math. 20 (1990), 285-316.

[Bu] C. Busquf́, Directly finite aleph-nought-complete regular rings are unit-regular, in Proceedings of the First International Meeting on Ring Theory, Granada, Spain 1986, J.L. Bueso, P. Jara and B. Torrecillas (eds.), Lecture Notes in Math. 1328, Springer-Verlag, Berlin-New York, 1988, pp. 38-49.

[E] G. A. ELLIOTT, Dimension groups with torsion, Internat. J. Math. 1 (1990), 361-380.

[G1] K. R. GOODEARL, "Von Neumann regular rings," Pitman, London, 1979.

[G2] K. R. GooneaRL, Directly finitc aleph-nought-continuous regular rings, Pacific J. Math. 100 (1982), 105-122.

[G3] K. R. GoODEARL, Metrically complete regular rings, Trans. Amer. Math. Soc. 272 (1982), 275-310.

[G4] K. R. GOODEARL, "Partially ordered abelian groups with interpolation," Math. Surveys and Monographs 20, Amer. Math. Soc., Providence, 1986.

[G5] K. R. GOODEARL, Unpublished notes on simple regular rings, 1990.

[GM] K. R. GOODEARL AND P. MENAL, Stable range one for rings with many units, J. Pure Applied Algebra 54 (1988), 261-287.

[GMM] K. R. Goodearl, P. Mennl and J. Monchsi, Frec and residually artinian regular rings, J. Algebra (to appear).

[M] J. Movcasi, Rang estable en anells regulars, Ph.D. Thesis, Lniversitat Autònoma de Barcelona, 1984.

[MM] P. MENAL AND J. MONCASI, On regular rings with stable range 2, J. Pure Applied Algebra 24 (1982), 2540.

[O] K. C. O'MfakA, Simple regular rings satisfying weak comparability, J. Algebro 141 (1991), 162186. 
[R] M. RIEFfEL, The canccllation Theorem for projective modules over irrational rotation $C^{*}$-algebras, Proc. London Math. Soc. 47 (1983), 285-302.

[V] L. N. VASERSTEIN, Stable rank of rings and dimensionality of topological spaces, Func. Anal. Applic. 5 (1971), 102-110.

[W] R. B. WARFIELD, JR., Cancellation of modules and groups and stable range of endomorphism rings, Pacific J. Math. 91 (1980), $457-485$.

Pere Ara:

Departament de Matemàtiques Universitat Autonoma de Barcelona 08193 Bellaterra (Barcelona)

SPAIN
K.R. Goodearl:

Department of Mathematics University of Utah

Salt Lake City

Utah 84112

U.S.A.

Rebut el 28 de Gener de 1992 